\title{
Fluid Driven by Tangential Velocity and Shear Stress: Mathematical Analysis, Numerical Experiment, and Implication to Surface Flow
}

\author{
H. S. Tang, ${ }^{1,2}$ L. Z. Zhang, ${ }^{1,3}$ J. P.-Y. Maa, ${ }^{4}$ H. Li, ${ }^{1}$ C. B. Jiang, ${ }^{2}$ and R. Hussain ${ }^{1}$ \\ ${ }^{1}$ Department of Civil Engineering, City College of New York, The City University of New York, 138th Street and Convent Avenue, \\ New York, NY 10031, USA \\ ${ }^{2}$ School of Hydraulic Engineering, Changsha University of Sciences and Technology, Changsha, Hunan 410114, China \\ ${ }^{3}$ Department of Applied Math, Shanghai University of Finance and Economics, Shanghai 200433, China \\ ${ }^{4}$ Virginia Institute of Marine Science, College of William and Mary, Gloucester Point, VA 23062, USA
}

Correspondence should be addressed to H. S. Tang; htang@ccny.cuny.edu

Received 18 November 2012; Revised 31 December 2012; Accepted 31 December 2012

Academic Editor: M. R. Hajj

Copyright (c) 2013 H. S. Tang et al. This is an open access article distributed under the Creative Commons Attribution License, which permits unrestricted use, distribution, and reproduction in any medium, provided the original work is properly cited.

\begin{abstract}
This paper investigates behaviors of flows driven by tangential velocity and shear stress on their boundaries such as solid walls and water surfaces. In a steady flow between two parallel plates with one of them in motion, analytic solutions are the same when a velocity and a shear stress boundary condition are applied on the moving plate. For an unsteady, impulsively started flow, however, analysis shows that solutions for velocity profiles as well as energy transferring and dissipation are different under the two boundary conditions. In an air-water flow, if either a velocity or a stress condition is imposed at the air-water interface, the problem becomes ill-posed because it has multiple solutions. Only when both of the conditions are specified, it will have a unique solution. Numerical simulations for cavity flows are made to confirm the theoretical results; a tangential velocity and a shear stress boundary condition introduce distinct flows if one considers an unsteady flow, whereas the two conditions lead to a same solution if one simulates a steady flow. The results in this paper imply that discretion is needed on selection of boundary conditions to approximate forcing on fluid boundaries such as wind effects on surfaces of coastal ocean waters.
\end{abstract}

\section{Introduction}

Flows driven by forcings at fluid boundaries are widely encountered, and they play important roles in various problems in nature and engineering applications. The boundaries can be air-water interfaces or solid walls, and examples of such flows include water currents in rivers, lakes, and oceans [1], water flows in settling tanks of wastewater treatment plants [2], and liquid flows in microelectronic devices [3]. Various types of forcings occur on fluid boundaries, and they directly affect the motion of the fluids. For instance, water receives wind stress and reacts to the atmosphere at ocean surfaces, or, air-water interfaces, on which a number of chemical and biological processes happen, and the resulting surface flows play a significant role in motion as well as quality of the whole water bodies in the oceans [4]. Therefore, it is crucially important to investigate fluid flows under different driving forces on fluid boundaries.

The physical forcings that a fluid experiences at its boundaries come from either another fluid or a solid wall, and they frequently appear as two types of boundary conditions: a tangential velocity and a shear stress condition $[5,6]$. For example, the moving lid of a cavity flow is a tangential velocity condition, and a wind blowing on surfaces of ocean currents can be a shear stress condition [5, 7]. Essentially, a velocity condition is a Dirichlet condition, and a shear stress condition is a Neumann condition.

Flows driven by both tangential velocity and shear stress have been previously analyzed. Analytical solutions for steady flows between two parallel plates such as the Couette flow can be found in literature [8]. Unsteady flows caused by a sudden movement of a plate at a constant or 
a time-dependent speed, namely, the Stokes' first and second problems, can be analytically solved [9-12]. Discussions on flows introduced by velocity boundary conditions have been extended to non-Newtonian fluids [13]. Flows under a shear stress condition have also been examined, and the existing work with this regard mainly focuses on practical problems with complicated geometries and numerical simulations [3, $14,15]$. However, literature on fundamental phenomena in flows driven by shear stress conditions is relatively sparse, and interestingly, to the best knowledge of the authors, no exact solutions have been reported for an impulsively started flow due to a shearing, although that for the flow under a tangential velocity condition, namely, the Stokes' first problem, has been available since a long time ago $[9,10]$.

Flows under action of velocity and shear conditions at their boundaries involve basic behaviours of fluids, such as mixing, diffusion, and energy transferring from the boundaries into their interiors, and a clear understanding of them will shed light on mechanism of more complicated flows. Although there have been a number of investigations on such flows, some issues related to their fundamental aspects are still unclear, such as the difference and similarity of the flows under these two conditions. As an example, in coastal ocean flow modelling, shear stress is commonly used at a boundary condition at water surfaces to reflect wind effects [7]. But, is it appropriate to impose a shear condition on the surfaces, or, can we specify a velocity condition instead? It is necessary to get answers for such questions since this will enable us to correctly study and understand many problems. The objective of this study is to clarify the difference and similarity in flow behaviors under a prescribed tangential velocity and a shear stress condition at fluid boundaries. We will study a few typical flows both analytically and numerically and examine their behaviours with respect to distributions and evolutions of velocity, stress, and energy.

The rest of this paper is organized as follows. Section 2 studies steady flows between two parallel plates with velocity and shear stress being imposed on one of them. In Section 3, an impulsively started flow under a shear condition is analytically solved and compared with the solution of its counterpart, the classic Stokes' first problem in which a velocity condition is imposed. An exact solution of an air-water transient flow is presented, and its uniqueness is discussed in Section 4. In order to illustrate the analyses presented in the previous sections for more complicated problems, numerical solutions are made for cavity flows in Section 5. Section 6 concludes this study with remarks on difference and similarity of flows under velocity and shear conditions and their implification to modelling of actual flows.

\section{Steady Flow Driven by Velocity and Stress Condition}

Consider an incompressible, fully developed, steady flow between two horizontal, parallel plates with gap $h$. The flow is driven by either a velocity or a shear stress at the top plate, and it has an upper layer moving to the right and a lower layer to the left, with zero net cross section flow rate. The momentum equation of the flow in the horizontal direction can be simplified as [8]

$$
-\frac{1}{\rho} \frac{d p}{d x}+v \frac{d^{2} u}{d y^{2}}=0
$$

here $x$ and $y$ are respectively the coordinates in the horizontal and the vertical direction, $u$ is the velocity in $x$ direction, $p$ is the pressure, $\rho$ is the density, and $v$ is the kinematic viscosity. When the pressure gradient is a constant, the solution of the horizontal velocity reads as

$$
-\frac{y^{2}}{2} \frac{d p}{d x}+\mu u+A y+B=0,
$$

where $\mu=\rho v$, being the dynamic viscosity, and $A, B=$ const. Imposing a velocity condition at the top plate, or $u=V_{0}$ at $y=h$, and the zero flow rate condition at the cross section, (2) can be solved as [8]

$$
u=3 V_{0} \frac{y^{2}}{h^{2}}-2 V_{0} \frac{y}{h} .
$$

Instead of the velocity condition at the top boundary, if we specify a shear stress at the top wall, or $\tau=\tau_{0}$ at $y=h$, the velocity is derived from (2) as

$$
u=\left(\frac{3}{4} \frac{y^{2}}{h}-\frac{y}{2}\right) \frac{\tau_{0}}{\mu},
$$

which leads to the following value of the velocity at the top boundary:

$$
\left.u\right|_{y=0}=\frac{\tau_{0} h}{4 \mu} .
$$

If we eliminate $\tau_{0}$ in (4a) with the aid of (4b) and let $\left.u\right|_{y=0}=$ $V_{0}$, it will be seen that the solution (4a) is actually same to solution (3). This indicates that, in case of a steady system, the solution of the flow driven by a velocity condition at the top plate is actually same to that forced by a shear stress condition (Figure 1). as

The power input from the top wall to the fluid is derived

$$
P=\tau_{0} V_{0}=\frac{4 \mu V_{0}^{2}}{h}
$$

and it is proportional to $V_{0}^{2}$ and inversely proportional to $h$. The viscous dissipation within the fluid is derived from solution (3) as [8]

$$
\Phi=\mu\left(\frac{d u}{d y}\right)^{2}=\left(\frac{3 y}{h}-1\right)^{2} \frac{4 \mu V_{0}^{2}}{h^{2}}
$$

which shows that it has a maximum at the top, gradually decreases to zero at $y=h / 3$, and then increases to another maximum at the bottom. 


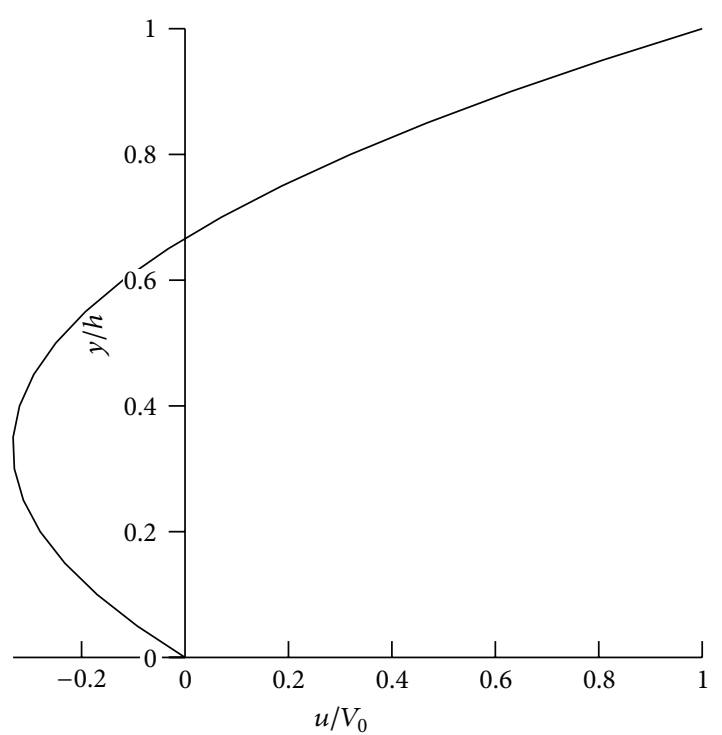

Figure 1: Solution (3) or, solution (4a) and (4b), for a steady flow between two plates. For this flow, a velocity and stress condition at $y / h=1$ lead to a same solution.

\section{Impulsively Started Flow under Velocity and Stress Condition}

Consider a fluid $(y>0)$ over an infinite, horizontal, and flat plate $(y=0)$. Initially the fluid is static, and then it flows suddenly because of application of a tangential velocity or a shear stress on surface of the plate. The governing equation for the flow is [8]

$$
\frac{\partial u}{\partial t}=v \frac{\partial^{2} u}{\partial y^{2}}
$$

and the associated initial and boundary conditions are

$$
\begin{aligned}
& u(y, 0)=0, \\
& u(0, t)=V_{0}, \\
& u(\infty, t)=0 .
\end{aligned}
$$

This is the classic Stokes' first problem, and its solution (see Figure 2(a)) is first given by Stokes and obtained again by Raylegh [9], which reads as [8]

$$
u(y, t)=V_{0}\left(1-\operatorname{erf}\left(\frac{y}{2 \sqrt{v t}}\right)\right)
$$

where $\operatorname{erf}(\cdot)$ is the error function.

Remark 1. Solution (9) is restricted with infinite depth of the fluid. A solution for a finite depth is given in [12].

If we specify a constant stress condition on the plate surface, governing equation (7), initial condition (8a), and

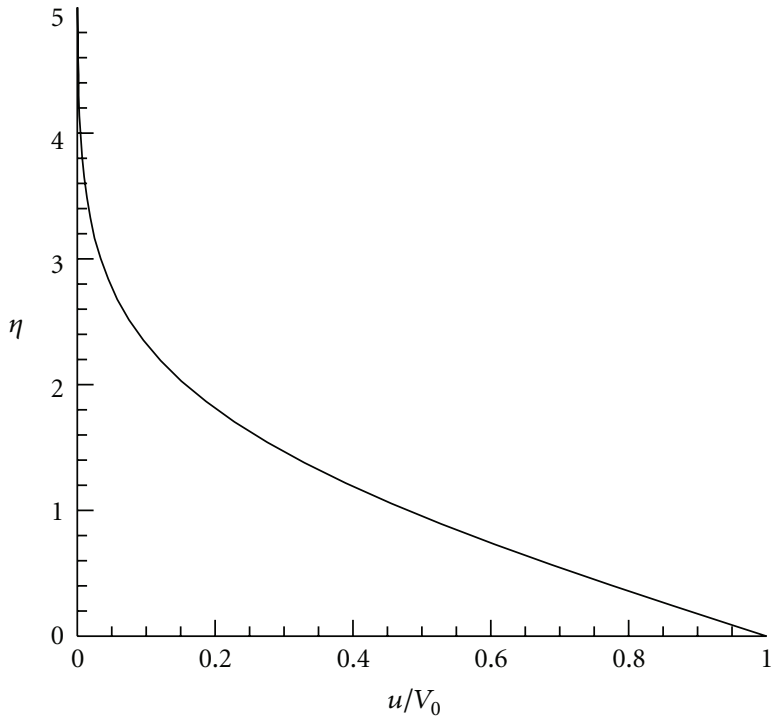

(a)

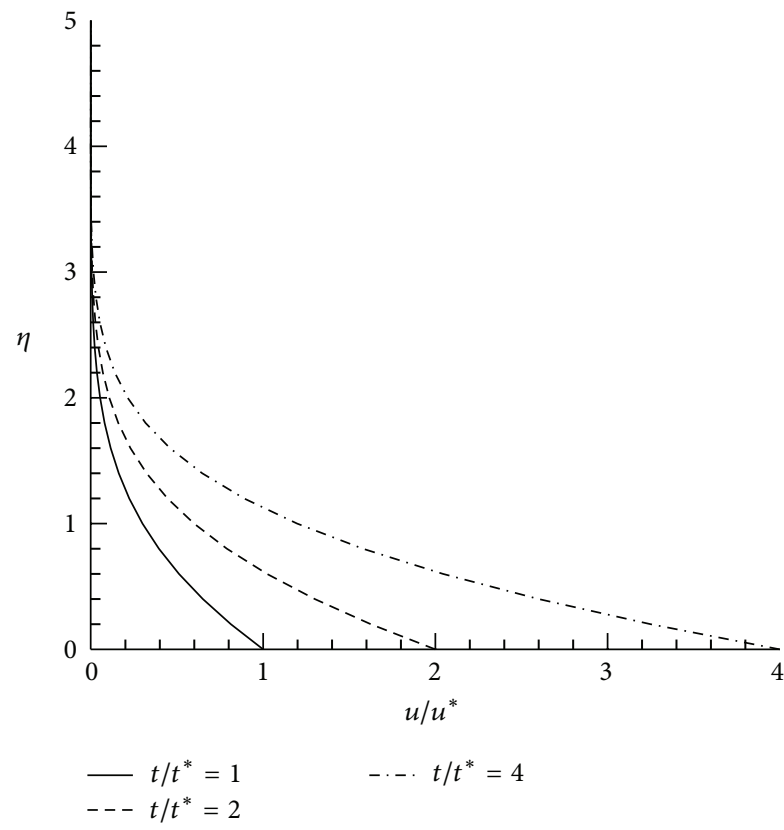

(b)

FIGURE 2: Solution for an impulsively started flow over a plate with (a) velocity boundary condition and (b) shear stress boundary condition. Here $t^{*}=\rho v / \tau_{0}, \eta=y / \sqrt{v t}$, and $u^{*}=\sqrt{\tau_{0} / \rho}$.

boundary condition (8c) remain the same, but (8b) will be replaced by the shear stress boundary condition as follows:

$$
\left.\frac{\partial u(y, t)}{\partial y}\right|_{y=0}=\frac{\tau_{0}}{\mu} .
$$

Taking Laplace transform of (7) over $t$, one has

$$
\frac{v}{s} \frac{\partial^{2} U(y, s)}{\partial y^{2}}-U(y, s)=0,
$$


where $s$ is a real number and $U(y, s)$ is the Laplace transform of $u(y, t)$. Equation (11) is solved as

$$
U(y, s)=A(s) \exp \left(-y \sqrt{\frac{s}{v}}\right)+B(s) \exp \left(y \sqrt{\frac{s}{v}}\right) .
$$

The boundary conditions (8c) and (10) can be transformed as

$$
\begin{gathered}
U(+\infty, s)=0, \\
\left.\frac{\partial U(y, s)}{\partial y}\right|_{y=0}=\frac{\tau_{0}}{\mu s},
\end{gathered}
$$

by which the constants in (12) are determined as

$$
A(s)=-\frac{\tau_{0}}{\mu s} \sqrt{\frac{\nu}{s}}, \quad B(s)=0 .
$$

Plugging (14) into (12) and taking inverse Laplace transform, one obtains the solution (see Figure 2(b)) for the flow with a stress boundary condition as follows:

$$
\begin{aligned}
u(y, t)= & -\frac{\tau_{0} y}{\rho v} \\
& \times\left[1-\operatorname{erf}\left(\frac{y}{2 \sqrt{v t}}\right)-\frac{2 \sqrt{v t}}{\sqrt{\pi} y} \exp \left(-\frac{y^{2}}{4 v t}\right)\right] .
\end{aligned}
$$

Using the velocity given in (9) to estimate the shear stress at the bottom wall, that is, $\tau=\mu d u / d y$ at $y=0$, the result is

$$
\left.\tau(t)\right|_{y=0}=-\frac{\mu V_{0}}{\sqrt{\pi v t}} .
$$

In addition, the shear stress at the bottom wall is obtained from (15) as

$$
\tau_{0}=-\frac{\mu \sqrt{\pi} u(0, t)}{2 \sqrt{v t}} .
$$

Equations (16) and (17) clearly indicate that solution (9) and (15) are different in physics; in the former, the shear stress at the wall will decrease with time and become zero as time approaches infinity, while the velocity at the bottom wall remains constant. Whereas, in the latter, the shear stress is a constant as the velocity keeps increasing with time. The power to maintain the former flow is

$$
P_{v}(t)=\frac{\mu V_{0}^{2}}{\sqrt{\pi v t}},
$$

and that for the latter is

$$
P_{s}(t)=\frac{2 \sqrt{v t} \tau_{0}^{2}}{\mu \sqrt{\pi}} .
$$

Therefore, the input power in the former decreases with time, while that in the latter it increases with time. When a velocity condition is applied, the viscous dissipation within the fluid is derived as

$$
\Phi_{v}=\frac{\rho V_{0}^{2}}{\pi t} e^{-\left(y^{2} / 2 v t\right)},
$$

and as a stress condition is specified, it becomes

$$
\Phi_{s}=\frac{\tau_{0}^{2}}{\rho}\left(1-\operatorname{erf}\left(\frac{y}{2 \sqrt{v t}}\right)\right)^{2} .
$$

With time, at any given $y$, viscous dissipation approaches zero in the former situation, while it approaches a constant in the latter case.

Remark 2. Equations (16)-(21) show clearly that an unsteady flow driven by a tangential velocity is distinct from that caused by a shear stress with regard to velocity profile, power, and viscous dissipation. Equation (17) shows the flow velocity at the surface will keep increasing with time, and this implies that a flow under a shear stress, such as a current in an open sea under action of a constant wind shear stress, will move faster and faster and cannot reach a steady state. Equation (19) tells that this flow is difficult to maintain since it requires that the amount of power input increases with time.

Remark 3. In the steady flow in Section 2, as indicated by (4b), (5), and (6), both power transferring and viscous dissipation are proportional to $V_{0}^{2}$ or $\tau_{0}^{2}$. It is seen in (18)(21) that this is also true in the impulsively started flows. In this sense, the impulsively started flows are consistent with the steady flow.

\section{Impulsively Started Air-Water Flow}

Consider a two-layer, air-water flow in the horizontal direction. Initially the air moves at a uniform velocity $V_{0}$, while the water is stationary. Both the air and the water have an infinite depth in the $y$ direction. The flow problem is formulated as follows:

$$
\begin{gathered}
\frac{\partial u}{\partial t}=v_{a} \frac{\partial^{2} u}{\partial y^{2}}, \quad y>0, \\
\frac{\partial u}{\partial t}=v_{w} \frac{\partial^{2} u}{\partial y^{2}}, \quad y<0, \\
u(y, 0)=0, \quad y<0, \quad u(y, 0)=V_{0}, \quad y>0, \\
u(+\infty, t)=V_{0}, \quad u(-\infty, t)=0, \\
u\left(0^{-}, t\right)=u\left(0^{+}, t\right), \\
\left.\mu_{w} \frac{\partial u(y, t)}{\partial y}\right|_{y=0^{-}}=\left.\mu_{a} \frac{\partial u(y, t)}{\partial y}\right|_{y=0^{+}},
\end{gathered}
$$

where $v_{a}$ and $v_{w}$ are the kinematic viscosity of air and water, respectively, and $\mu_{a}$ and $\mu_{w}$ are the dynamic viscosity of air and water, respectively. In this case, match conditions (22e) and (22f) for both velocity and stress are applied at the airwater interface, that is, at $y=0$. In other words, velocity and shear stress are continuous at the interface. 
Using Laplace transform for the partial differential equation (22a), the resulting equation can be solved as

$$
\begin{aligned}
U(y, s)= & A^{+}(s) \exp \left(y \sqrt{\frac{s}{v_{a}}}\right) \\
& +B^{+}(s) \exp \left(-y \sqrt{\frac{s}{v_{a}}}\right)+\frac{V_{0}}{s}, \quad y>0 .
\end{aligned}
$$

According to boundary condition (22d), we have

$$
U(+\infty, s)=\frac{V_{0}}{s},
$$

which implies that $A^{+}(s)$ must be zero. Similarly, the solution for the water flow in the transformed space is derived from $(22 \mathrm{~b})$ as

$$
\begin{aligned}
U(y, s)= & A^{-}(s) \exp \left(y \sqrt{\frac{s}{v_{w}}}\right) \\
& +B^{-}(s) \exp \left(-y \sqrt{\frac{s}{v_{w}}}\right), \quad y<0 .
\end{aligned}
$$

From boundary condition (22d), it is derived that

$$
U(-\infty, s)=0,
$$

which requires that $B^{-}(s)$ be zero. Using Laplace transform, the match conditions (22e) and (22f) can be converted into

$$
\begin{gathered}
U\left(0^{-}, s\right)=U\left(0^{+}, s\right), \\
\left.\mu_{a} \frac{\partial U(y, s)}{\partial y}\right|_{y=0^{+}}=\left.\mu_{w} \frac{\partial U(y, s)}{\partial y}\right|_{y=0^{-}},
\end{gathered}
$$

combining solutions (23) and (25) with the above equations, one has

$$
\begin{aligned}
A^{-}(s) & =\frac{\mu_{a} \sqrt{v_{w}}}{\mu_{a} \sqrt{v_{w}}+\mu_{w} \sqrt{v_{a}}} \frac{V_{0}}{s}, \\
B^{+}(s) & =-\frac{\mu_{w} \sqrt{v_{a}}}{\mu_{a} \sqrt{v_{w}}+\mu_{w} \sqrt{v_{a}}} \frac{V_{0}}{s} .
\end{aligned}
$$

Plugging (28) into (23) and (25) and then taking inverse Laplace transform, the solution of problem (22a)-(22f) is derived as

$$
\begin{aligned}
& u(y, t) \\
& =\left\{\begin{array}{r}
V_{0}+\frac{V_{0}}{1+\left(\mu_{a} / \mu_{w}\right) \sqrt{v_{w} / v_{a}}}\left(\operatorname{erf}\left(\frac{y}{2 \sqrt{v_{a} t}}\right)-1\right), \\
\frac{V_{0}}{1+\left(\mu_{w} / \mu_{a}\right) \sqrt{v_{a} / v_{w}}}\left(1-\operatorname{erf}\left(\frac{-y}{2 \sqrt{v_{w} t}}\right)\right), \\
y<0
\end{array}\right.
\end{aligned}
$$

which is plotted in Figure 3. It can be proven that (29) is a unique solution for problem (22a)-(22f) (see the Appendix).

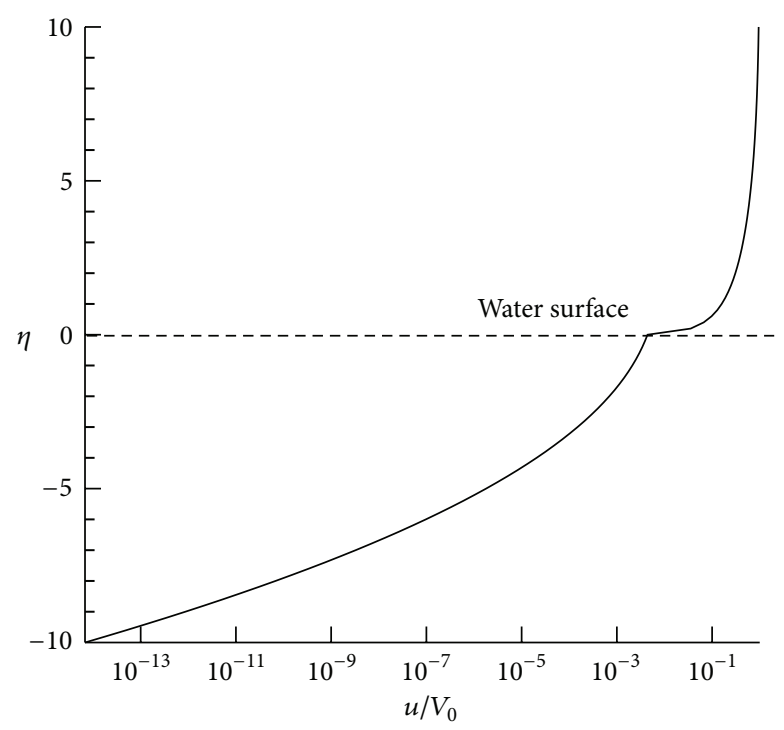

FIGURE 3: Solution (29) for air-water flow problem (22a)-(22f): air and water at $15^{\circ} \mathrm{C}$ with $\mu_{a}=1.79 \times 10^{-5} \mathrm{~N} \cdot \mathrm{s} / \mathrm{m}^{2}, v_{a}=1.46 \times 10^{-5} \mathrm{~m}^{2} / \mathrm{s}$ for air, $\mu_{w}=1.12 \times 10^{-3} \mathrm{~N} \cdot \mathrm{s} / \mathrm{m}^{2}$, and $v_{w}=1.12 \times 10^{-6} \mathrm{~m}^{2} / \mathrm{s}$ for water. Here $\eta=y / \sqrt{v_{w} t}$.

Remark 4. Equation (29) is not restricted for air-water flow, and it can be used for any other two fluids. It is applicable if the thickness of lower and upper layers is large, and the flow is free from the effect of walls. Analytical solutions considering finite depth of air and water have been presented in [12].

Velocity presented by solution (29) is discontinuous in both $y$ and $t$ when $t=0$. When $t>0$, the velocity is continuous, and, interestingly, it is a constant at the air-water interface:

$$
\frac{u(0, t)}{V_{0}}=\frac{1}{1+\left(\mu_{w} / \mu_{a}\right) \sqrt{v_{a} / v_{w}}} .
$$

For the flow in Figure 3, the velocity at the interface, $u(0, t)$, or the maximum of velocity in the water layer, is about $V_{0} / 227$. The power transferred from the air to the water is

$$
P_{a-w}(t)=\frac{\mu_{a}^{2} \mu_{w} V_{0}^{2}}{2\left(\mu_{w} \sqrt{v_{a}}+\mu_{a} \sqrt{v_{w}}\right)^{2}} \sqrt{\frac{v_{w}}{t}} .
$$

The viscous dissipation is derived as

$$
\begin{aligned}
& \Phi_{a-w} \\
& = \begin{cases}\frac{V_{0}^{2}}{\left(1+\left(\mu_{a} \sqrt{v_{w}}\right) \sqrt{v_{w} / v_{a}}\right)^{2}} \frac{1}{\pi v_{a} t} \exp \left(-\frac{y^{2}}{2 v_{a} t}\right), & y>0, \\
\frac{V_{0}^{2}}{\left(1+\left(\mu_{w} \sqrt{v_{a}}\right) \sqrt{v_{a} / v_{w}}\right)^{2}} \frac{1}{\pi v_{w} t} \exp \left(-\frac{y^{2}}{2 v_{w} t}\right), & y<0 .\end{cases}
\end{aligned}
$$


Remark 5. In coastal ocean flow modelling, it is common to include wind effects at air-water surfaces, or water surfaces, as a condition for water velocity as follows:

$$
\left.\rho_{w} K \frac{\partial u(y, t)}{\partial y}\right|_{y=0^{-}}=\tau_{a-w}
$$

and the air-water interface stress can be determined from the air flow using $\tau_{a-w}=\rho_{a} C_{d}\left[V_{10}-u(0, t)\right]^{2} / 2[19,20]$. Here, $\rho_{a}$ and $\rho_{w}$ are the air and water density, respectively, $K$ is the eddy viscosity coefficient, $C_{d}$ is the drag coefficient, and $V_{10}$ is the wind speed at $10 \mathrm{~m}$ above the water surface. This method to include wind effect is essentially the same as imposing the shear stress condition (10) to (7) or (22f) to (22b). Application of (33) in coastal ocean flow modelling and imposing the stress conditions (7) and (22f) could introduce same flow patterns, although the former could involve more complex phenomena such as turbulence and wave effects.

Here we post a question whether using the shear stress condition (33) to approximate wind effect at water surface is appropriate. First, (30) shows that, when a water body is driven by an air flow, the speed at their interface is a constant. But as indicated in Remark 2, if a shear stress condition is applied at the interface, the water will keep accelerating rather than moving at a constant speed. The water flow is difficult to maintain because it requires more and more amount of power supply, and this situation cannot happen in reality. Second, the trend in power transferred from the air to the water at the air-water surface, as shown in (31), is similar to that from the fluid surface into the fluid for the flow caused by a specified velocity boundary condition, see (18), and both of them are proportional to $V_{0}^{2}$ and inversely proportional to $\sqrt{t}$ (the former is much smaller, and $P_{a-w}(t) / P_{v}(t) \approx 0.000017$ for air and water at $15^{\circ} \mathrm{C}$ ). Third, from (32) it is seen that, with regard to $t$ and $V_{0}$, the evolution of the dissipation in water in the air-water flow is similar to that in the flow driven by a surface velocity boundary condition, see (20). In summary, the behaviors of a water flow driven by a surface velocity boundary condition are closer to those of a water flow moved by a layer of air.

However, if only one coupling condition is given at the air-water interface, either the velocity matching condition (22e) or the shear stress matching condition (22f), the problem described above will be ill-posed. This can be shown as follows. Let both $w_{1}$ and $w_{2}$ be a solution to problem (22a)(22e), in which only the condition for velocity match is used, and $w=w_{1}-w_{2}$. It can be shown that $w$ will be a solution of the following problem:

$$
\begin{gathered}
\frac{\partial w}{\partial t}=v_{w} \frac{\partial^{2} w}{\partial y^{2}}, \quad y<0, \\
\frac{\partial w}{\partial t}=v_{a} \frac{\partial^{2} w}{\partial y^{2}}, \quad y>0, \\
w(y, 0)=0, \quad-\infty<y<\infty, \\
w(+\infty, t)=0, \quad w(-\infty, t)=0, \\
w\left(0^{-}, t\right)=w\left(0^{+}, t\right) .
\end{gathered}
$$

Following the same procedure for obtaining (29), one can derives a solution in form of (23) and (25) in the Laplace transformed space of (34a)-(34e) together with the following conditions:

$$
\begin{gathered}
A^{+}(s)=B^{-}(s)=0, \\
A^{-}(s)=B^{+}(s)=f(s),
\end{gathered}
$$

where $f(s)$ can be any continuous function. If we let

$$
f(s)=\frac{c}{s},
$$

where $c$ is a nonzero constant, then the solution to (34a)(34e) becomes

$$
w(y, t)= \begin{cases}c\left(1-\operatorname{erf}\left(\frac{y}{2 \sqrt{\nu_{a} t}}\right)\right), & y>0, \\ c\left(1-\operatorname{erf}\left(-\frac{y}{2 \sqrt{\nu_{w} t}}\right)\right), & y<0 .\end{cases}
$$

If $c \neq 0, w(y, t)$ will not be zero, that is to say that problem (34a)-(34e) has a nonzero solution. Consequently, problem (22a)-(22e) will not have a unique solution. In this case, or (22f) is taken away, a solution for problem $(22 \mathrm{a})-(22 \mathrm{e})$ can be derived as

$$
u(y, t)= \begin{cases}V_{0}+c^{\prime} V_{0}\left(\operatorname{erf}\left(\frac{y}{2 \sqrt{\nu_{a} t}}\right)-1\right), & y>0, \\ \left(c^{\prime}-1\right) V_{0}\left(\operatorname{erf}\left(-\frac{y}{2 \sqrt{v_{w} t}}\right)-1\right), & y<0,\end{cases}
$$

where $c^{\prime} \in R$ and $c^{\prime} \neq 0$.

Similarly, it can be shown that problem (22a)-(22d) and (22f) will not have a unique solution if velocity condition (22e) is removed, and a solution is

$$
u(y, t)= \begin{cases}V_{0}+c^{\prime \prime} V_{0}\left(\operatorname{erf}\left(\frac{y}{2 \sqrt{\nu_{a} t}}\right)-1\right), & y>0, \\ c^{\prime \prime} \frac{\mu_{a} \sqrt{\nu_{w}}}{\mu_{w} \sqrt{\nu_{a}}} V_{0}\left(1-\operatorname{erf}\left(-\frac{y}{2 \sqrt{\nu_{w} t}}\right)\right), & y<0\end{cases}
$$

where $c^{\prime \prime} \in R$ and $c^{\prime \prime} \neq 0$. 


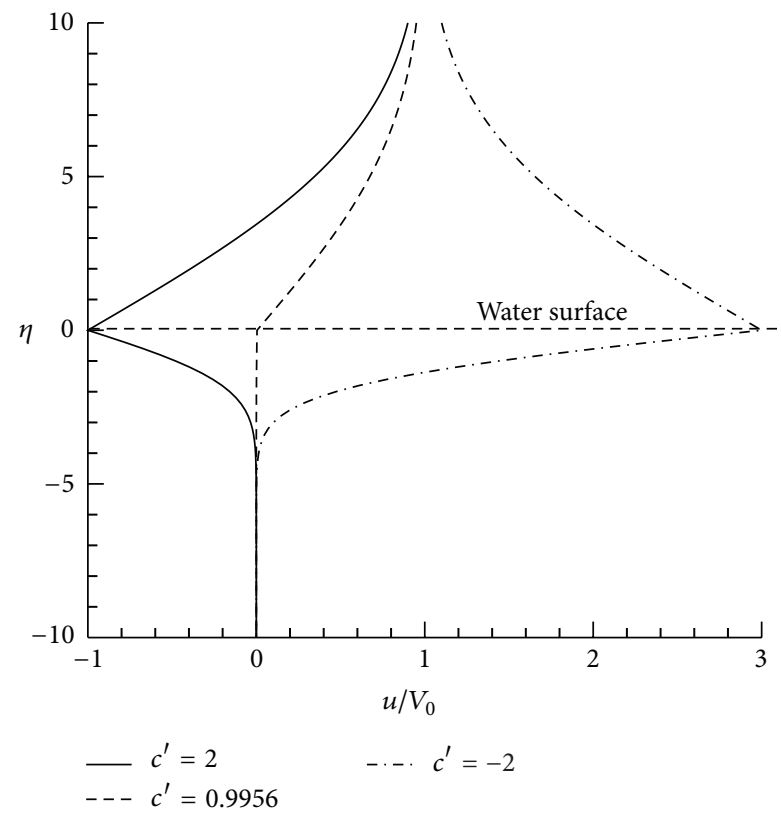

(a)

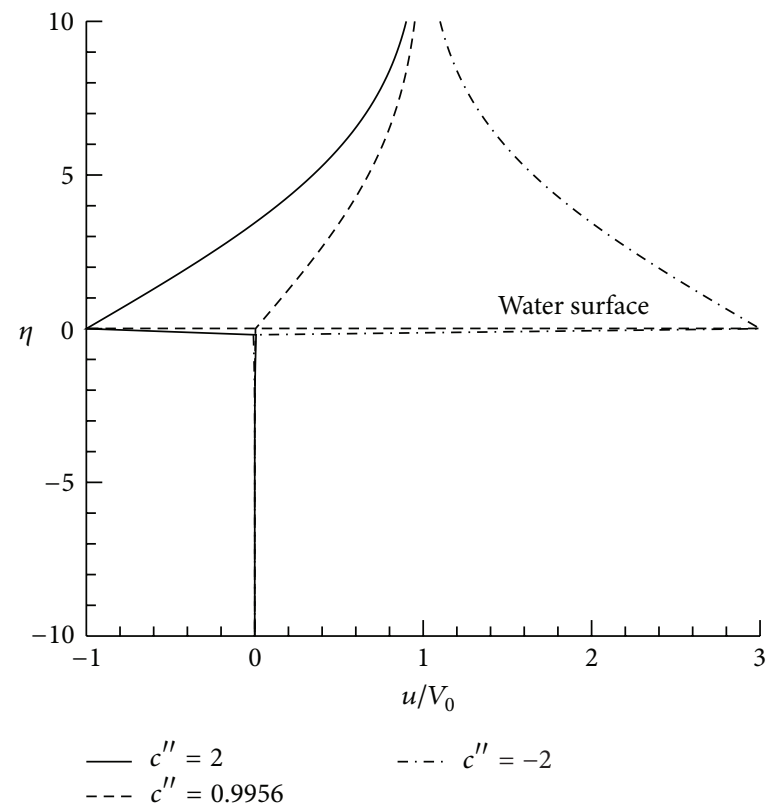

(b)

FIGURE 4: Multiple solutions for the air-water flow of (a) with only the velocity condition, that is, (38) and (b) with only the stress condition at the interface, that is, (39).

Solutions (38) and (39) with different values for $c^{\prime}, c^{\prime \prime}$ are shown in Figure 4. Equations (38) and (39) will become the true solution (29) when

$$
c^{\prime}=c^{\prime \prime}=\frac{1}{1+\left(\mu_{a} / \mu_{w}\right) \sqrt{v_{w} / v_{a}}},
$$

which equals 0.9956 for air and water at $15^{\circ} \mathrm{C}$.

Nonuniqueness of the solutions for the air-water flow (38) and (39) leads to a question: which solution is physically possible among the multiple solutions? According to the momentum principle, which all physical solutions should satisfy, it is derived that any solution of the flow is restricted by the following condition:

$$
\begin{aligned}
\int_{0}^{+\infty} \rho_{a} V_{0} d y= & \int_{0}^{+\infty} \rho_{a} u(y, t) d y \\
& +\int_{-\infty}^{0} \rho_{w} u(y, t) d y,
\end{aligned}
$$

which requires that the total momentum of the flow remains the same as the initial momentum. Plugging solution (38) into above condition, one has

$$
\begin{gathered}
\left(\rho_{a} V_{0} c^{\prime} \sqrt{v_{a} t}+\rho_{w} V_{0}\left(c^{\prime}-1\right) \sqrt{v_{w} t}\right) \\
\times \int_{0}^{+\infty}(1-\operatorname{erf}(x)) d x=0
\end{gathered}
$$

which leads to

$$
c^{\prime}=\frac{1}{1+\left(\mu_{a} / \mu_{w}\right) \sqrt{v_{w} / v_{a}}} .
$$

Under this requirement, solution (38) becomes (29) or the unique solution obtained when both the velocity and stress condition are applied. This shows that the restriction (41) selects (29) as the only solution, and it excludes others as possible solutions. However, following the same steps, it can be shown that the multiple solutions expressed in (39), or the solution associated with the only stress condition, can meet the condition (41), or, this condition cannot identify (29) as the only possible solution.

Remark 6. Continued on Remark 5, the conclusion that the air-water flow problem is well-posed only if both velocity and shear condition are applied further raises more concern on only using a stress condition to include wind effects in coastal ocean flow modelling. In addition, the results in this section indicate that when both velocity and stress connection conditions are applied at the interfaces, the velocity at the interface is a constant, as shown in (30). But, the stress there will be time dependent, which can be evaluated from the velocity given in (29), and it cannot be approximated as a constant on a water surface.

Remark 7. It is interesting that above analysis shows that integral form of momentum equation (41) can pick up a unique solution among these expressed in (38) but fails to do so for those expressed in (39). Therefore, a more universal condition is needed to identify a unique solution, or a so called "physical solution", among multiple solutions expressed in (38) and (39). 


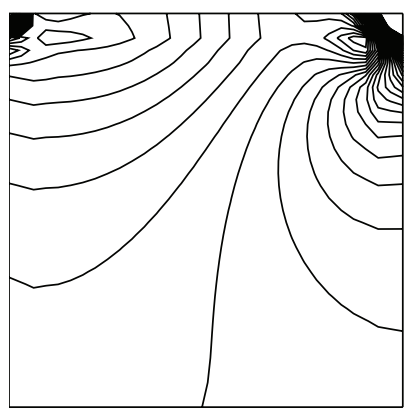

Pressure

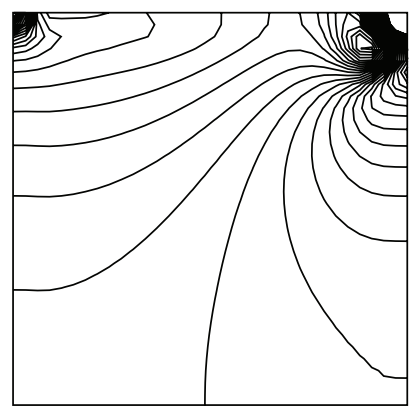

Pressure

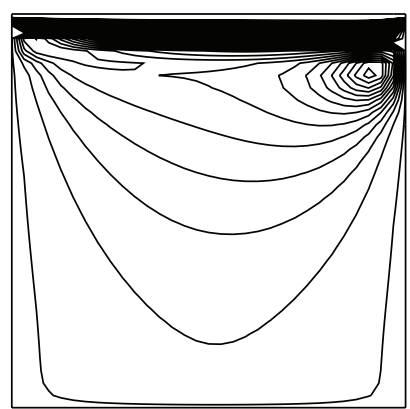

$u$-velocity

(a)

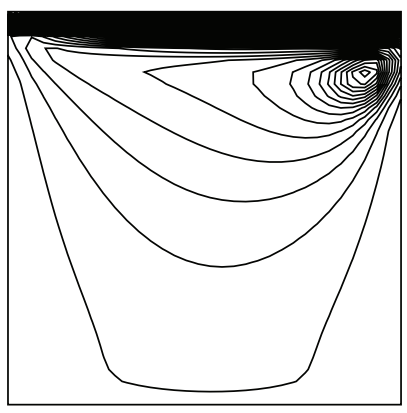

$u$-velocity

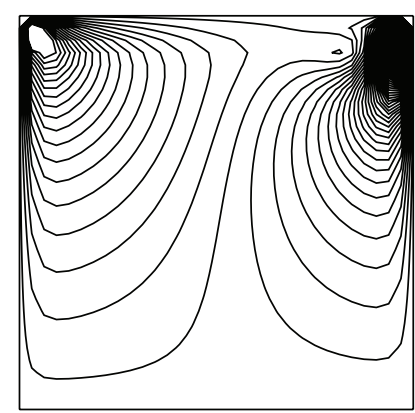

$w$-velocity

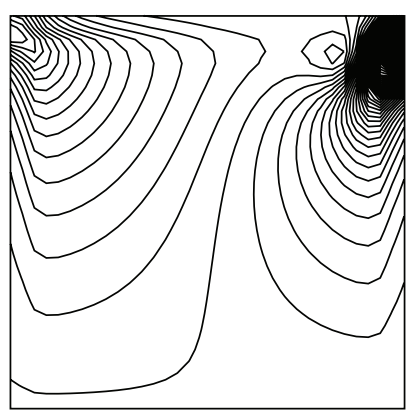

$w$-velocity

(b)

FIGURE 5: Solution for the cubic cavity flow, $\mathrm{Re}=1000$. (a) Solution obtained with the algorithm in [16]; (b) Solution given by FLUENT.

\section{Numerical Example}

In order to extend the theoretical studies for the purely shear flows presented in earlier sections to more complex situations, computer modelling is made to simulate the flow field of cavity flows, which are typically driven either by velocity or stress at their top walls [5]. A cavity flow exhibits various phenomena that can possibly occur, such as eddies, secondary flows, and complex three-dimensional (3D) patterns $[5,6]$. Therefore, it is an ideal test case and thus is selected to demonstrate the extension.

FLUENT is a commercially available numerical model that solves the Navie-Stokes equations. It has been successfully used to simulate various flows [21]. As a calibration, however, FLUENT is first employed to simulate a lid-driven, laminar, 3D, steady, cubic cavity flow. The flow is driven by a velocity boundary condition at the top wall. A uniform mesh with size $69 \times 69 \times 69$ is used, and the computation finishes in 124 seconds on a desktop. The simulation shows that the solution matches well with another solution obtained by a different model (Figure 5).

After the calibration, the FLUENT is used to simulate a wind-driving turbulent water flow in a laboratory flume [17]. The flume has a dimension of length $=30 \mathrm{ft}$, width $=3 \mathrm{ft}$, and water depth $=1 \mathrm{ft}$, which are set in direction of $x, y$, and $z$, respectively. The origins of $x$ and $y$ are selected at the center of the flume, and that of $z$ is at its bottom. The experimental condition is quantified by the shear velocity Reynolds number, $\operatorname{Re}=u^{*} d / v_{w}=1600$, with $u^{*}=\sqrt{\tau_{0} / \rho_{w}}$ and $d$ being the depth of the water. At $15^{\circ} \mathrm{C}$, $\rho_{w}=998.9 \mathrm{~kg} / \mathrm{m}^{3}$. Therefore, it is determined that $u^{*}=5.88 \times$ $10^{-3} \mathrm{~m} / \mathrm{s}$, and water surface shear stress $\tau_{0}=0.00345 \mathrm{~Pa}$. Using the value for $u^{*}$ and the experimental data in [17], it is determined that $V_{0}=0.1 \mathrm{~m} / \mathrm{s}$. The parameters selected for the cubic cavity flow and the standard $k-\varepsilon$ turbulent model are employed. The flume flow is treated as a long cavity flow, and the simulation of the wind-induced flow starts with a stationary water inside the cavity imposed with either a constant velocity boundary condition $\left(V_{0}=0.1 \mathrm{~m} / \mathrm{s}\right)$ or a constant shear stress boundary condition $\left(\tau_{0}=0.00345 \mathrm{~Pa}\right)$ on top of the cavity. Computations using two meshes $125 \times$ $34 \times 35$ and $251 \times 67 \times 71$ are employed. Mesh nodes are densely clustered near the six faces of the long cavity. The time step 1 second is used, and it takes 4 hours and 10 minutes and 7 hours and 52 minutes to finish the computation of the flow up to 500 seconds on the desktop for the coarse and fine mesh, respectively. The computational results show that the solution on the latter mesh can be considered mesh independent.

An instantaneous solution during the transient stage obtained with the velocity condition and another with the shear stress boundary condition are shown in Figure 6. As we can understand, it is seen that the velocity and stress conditions introduce flows different in velocity magnitudes. At this moment, when the velocity condition is applied, the velocity and stress magnitudes in the cavity are larger than those when the shear stress condition is applied. A quantification of the 


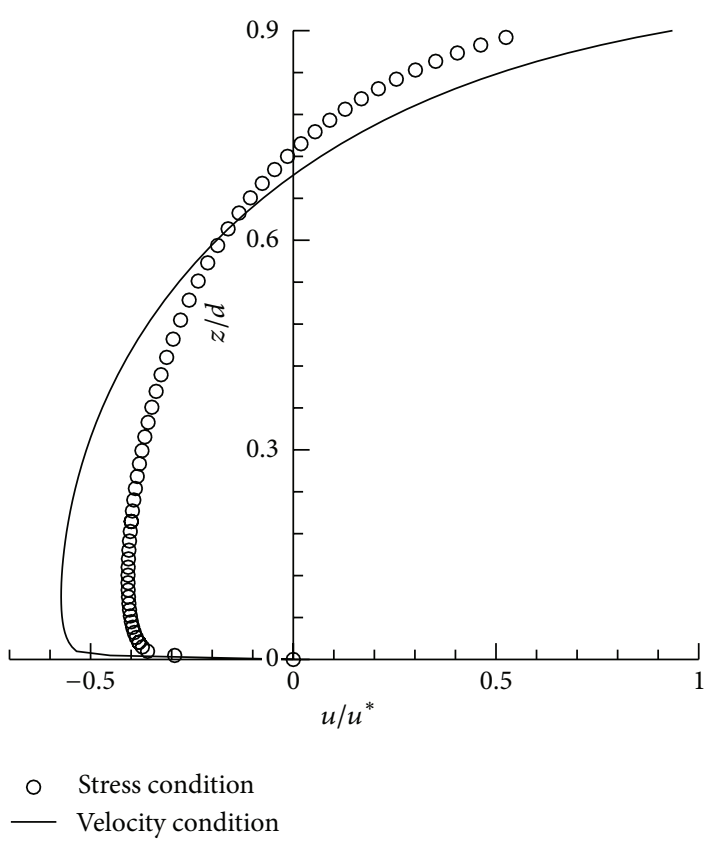

(a)

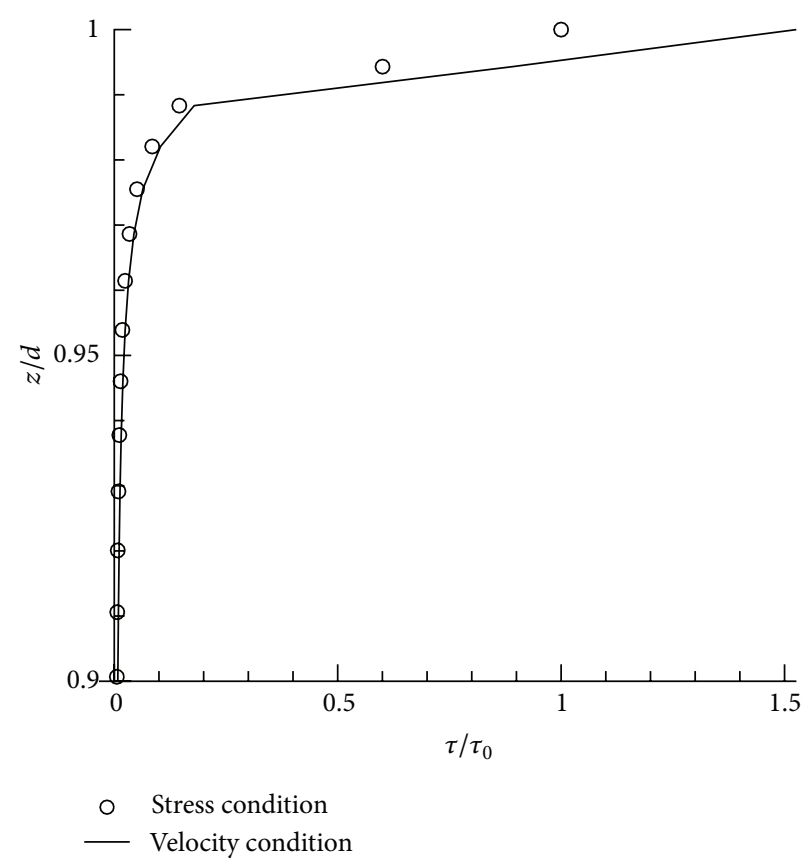

(b)

Figure 6: Velocity and shear stress distribution of the long cavity flow at $x=0, y=0$, and $t=50 \mathrm{~s}$.

difference between the solutions associated with the velocity and stress condition at this moment is given in Table 1.

Nevertheless, as time develops the solutions with the both boundary conditions tend to a steady state. At the steady state, the two solutions are indistinct (Figures 7 and 8). This is further illustrated by the computed difference of the two solutions in Table 1 . At the steady state, on the plane $y=0$, there is a limit point at the left side and a limit circle at the right end. At $x=14.5$, the flows have a divergence point near the top boundary and a convergence point near the bottom. It should be noted that in Figure 8 the velocity and stress profiles are very different from those of the analytical solution obtained in Section 2 for a steady flow between two infinite plates. The discrepancy between them is attributed to the fact that the former involve effects of wall and turbulence. As an evidence for the correctness of the simulation, the computed velocity profiles are plotted against the results from other approaches in Figure 9.

These numerical experiments extend the conclusions obtained with the analytical approaches in the previous sections: velocity and stress boundary conditions introduce distinct flows in case of unsteady flows, whereas, in contrast, they present same solutions for steady flows. It should be pointed out there is a seemly contradiction between a conclusion in Section 2 and numerical results in this section; while, as indicated in Remark 2, the surface velocity of a stressdriven flow with an infinite depth will keep increasing and the flow cannot reach a steady flow, the numerical solution for the top velocity of the flume flow in this section tends to a constant and the flow reaches a steady state as time goes. This is because that in the latter, the water depth is finite and the flow is restricted by its walls, and thus its surface velocity cannot keep increasing with time. Actually, given the limited depth and width, if the surface velocity tends to infinity, the shear stress at the top will also reach infinity, which violates the boundary condition of a constant shear stress at the top wall.

\section{Concluding Remarks}

This paper studies flows driven by velocity and stress conditions on fluid boundaries. It presents analytical solutions for a steady flow between two parallel plates, an impulsively started flow due to a constant shear stress, and an unsteady unidirection air-water flow. By analysis as well as numerical simulation, it is concluded that velocity and stress conditions applied at fluid boundaries produce flows with distinct velocity profiles, stress distributions, and energy fluxes in unsteady flows, whereas the two conditions lead to a same solution in steady flows. For an air-water flow, the problem has multiple solutions if a velocity condition alone or a stress condition alone is applied at the interface, and its solution is unique only when both a velocity and a shear stress condition are specified. It is also expected that the analytical solutions newly developed in this paper, such as (15) and (29), will be valuable tools for analysis and practical applications.

It is common to apply a shear stress condition on water surfaces to reflect wind effects in engineering applications such as coastal ocean modelling, but little literature is found on discussion on appropriateness of this treatment. Although this paper deals with purely shear flows and actual coastal ocean flows are much more complicated, the former are a fundamental form of the latter, and therefore its study has implication to actual problems. This paper shows that 


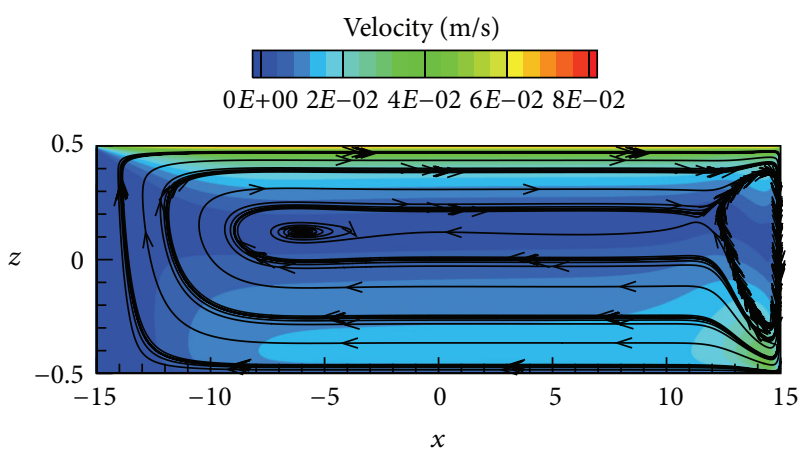

(a)

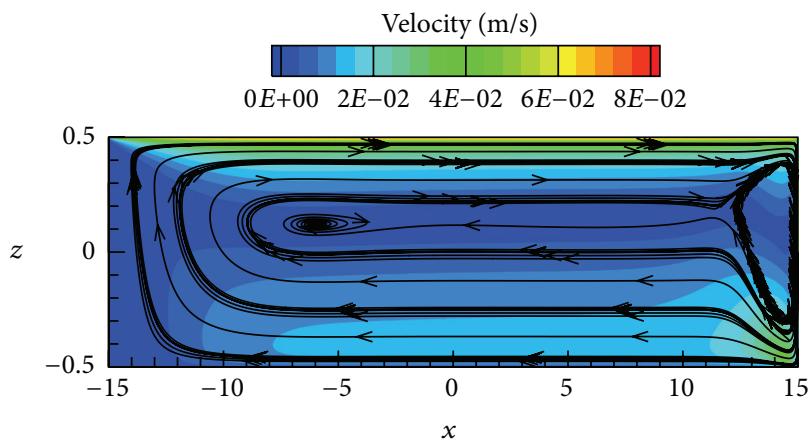

(c)

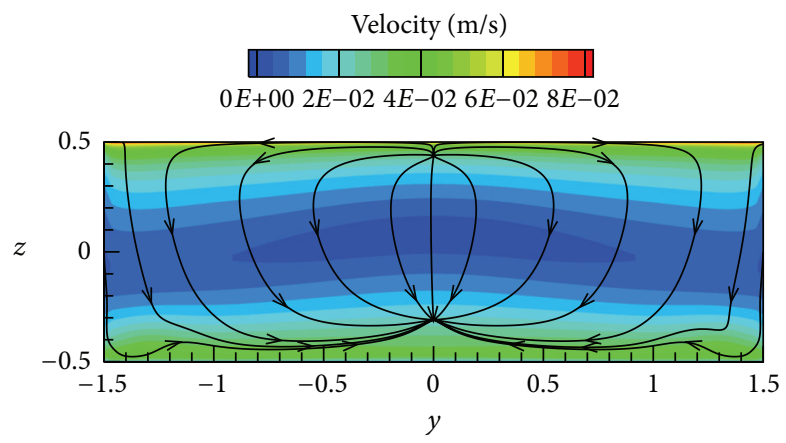

(b)

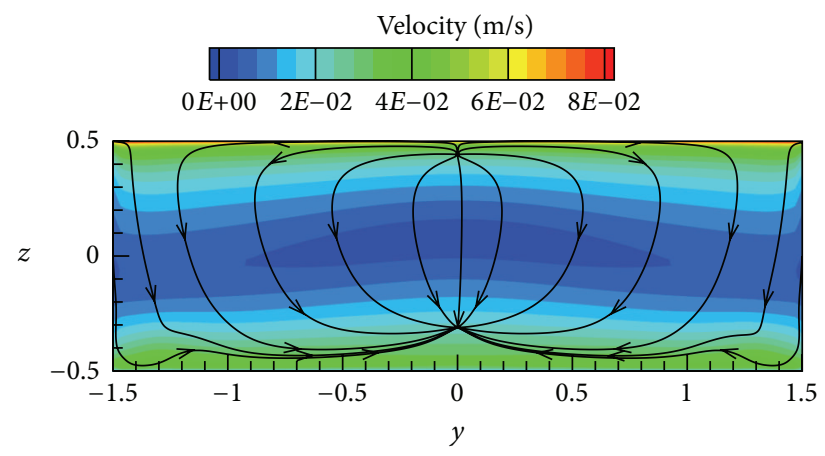

(d)

FIGURE 7: Velocity magnitude and streamlines of the long cavity flow at $t=500 \mathrm{~s}$, approximately the steady states, obtained by (a), (b), the velocity condition, and (c), (d) the shear stress condition. In (a) and (c), $y=0$, and in (b) and (c), $x=14.5$.

TABLE 1: The difference of solutions for the long cavity flow associated with the velocity and stress boundary condition. $u, v$, and $w$ are velocity in $x, y$, and $z$ direction, respectively. Subscripts $v$ and $s$ stand for the velocity and stress condition, respectively.

\begin{tabular}{lcccc}
\hline$t(\mathrm{~s})$ & $\left\|p_{s}-p_{v}\right\|_{\infty}(\mathrm{Pa})$ & $\left\|u_{s}-u_{v}\right\|_{\infty}(\mathrm{m} / \mathrm{s})$ & $\left\|v_{s}-v_{v}\right\|_{\infty}(\mathrm{m} / \mathrm{s})$ & $\left\|w_{s}-w_{v}\right\|_{\infty}(\mathrm{m} / \mathrm{s})$ \\
\hline 50 & 0.641 & 0.056 & $1.66 E-3$ & 0.0121 \\
500 & $4.30 E-3$ & $9.42 E-5$ & $1.51 E-4$ & $8.95 E-5$ \\
\hline
\end{tabular}

a velocity and a stress condition lead to different flows in presence of unsteadiness that is usually true in coastal flows. If only one of them is applied, as discussed in Remark 5 and Remark 6, the resulting flow becomes unsound in physics and the problem turns out to be ill-posed. Therefore discretion is needed on selection of boundary conditions on water surfaces, and a revisit could be necessary for the treatment of wind effects on water currents in their computer modelling.

\section{Appendix}

\section{Uniqueness of Solution to Problem (22a)-(22f)}

Let $w=w_{1}-w_{2}$, both $w_{1}$ and $w_{2}$ being a solution to problem (22a)-(22f). Then, it can be shown that $w$ will be a solution of the following problem:

$$
\frac{\partial w}{\partial t}=v_{a} \frac{\partial^{2} w}{\partial y^{2}}, \quad y>0
$$

$$
\begin{gathered}
\frac{\partial w}{\partial t}=v_{w} \frac{\partial^{2} w}{\partial y^{2}}, \quad y<0 \\
w(y, 0)=0, \quad y<0, \quad w(y, 0)=0, \quad y>0 \\
w(+\infty, t)=0, \quad w(-\infty, t)=0 \\
w\left(0^{-}, t\right)=w\left(0^{+}, t\right) \\
\left.\mu_{w} \frac{\partial w}{\partial y}\right|_{y=0^{-}}=\left.\mu_{a} \frac{\partial w}{\partial y}\right|_{y=0^{+}}
\end{gathered}
$$

If the solution to problem (Ala)-(Alf) only has zero as its solution, then problem $(22 \mathrm{a})-(22 \mathrm{f})$ has a unique solution. With the aid of the Laplace transform and boundary condition (Ald), one derives the solution for the above problem in the transformed space as

$$
\begin{array}{ll}
W(y, s)=B(s) e^{-y \sqrt{s / v_{a}}}, & y>0, \\
W(y, s)=A(s) e^{y \sqrt{s / v_{w}}}, & y<0,
\end{array}
$$




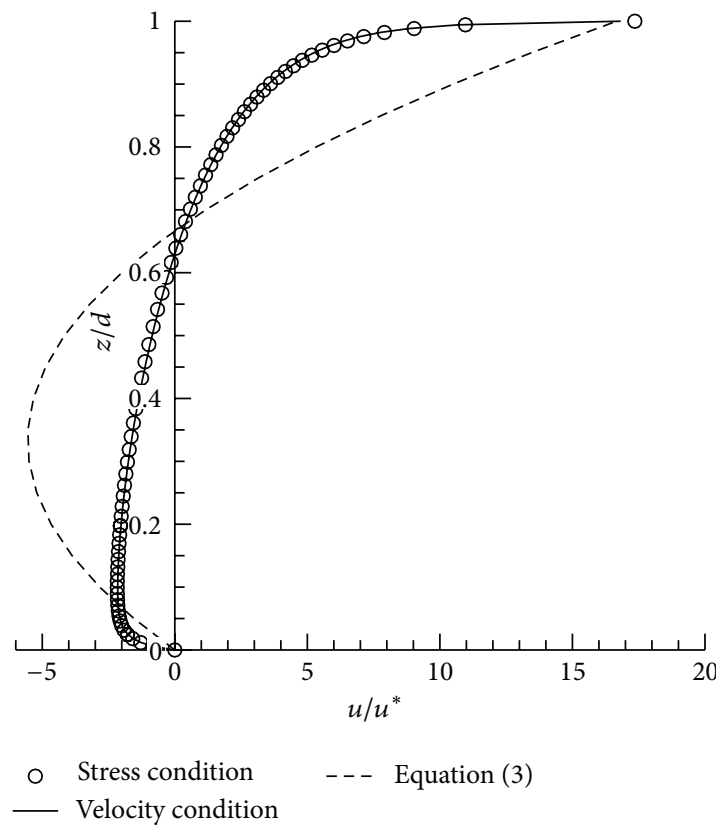

(a)

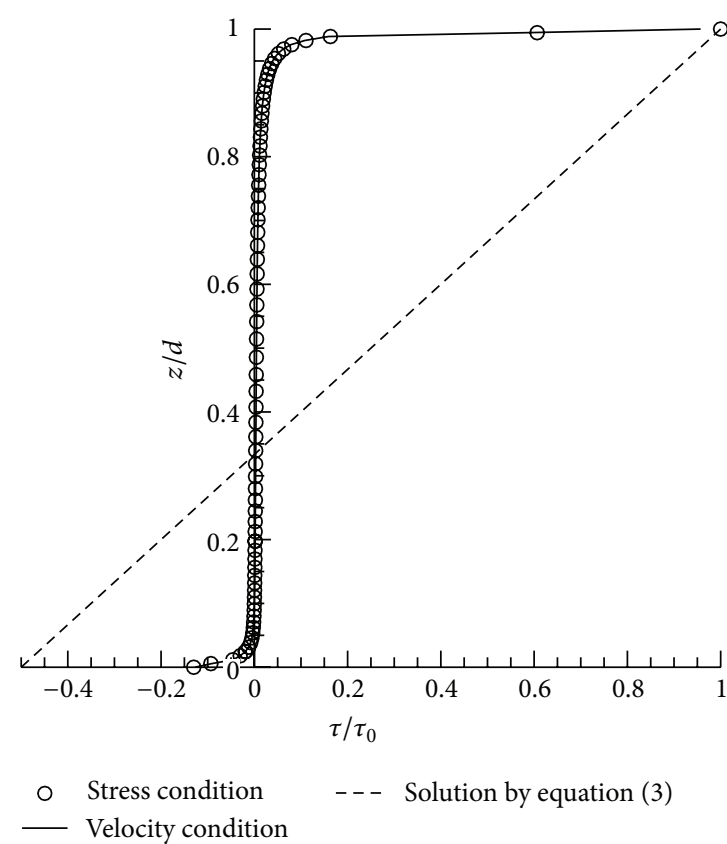

(b)

FIGURE 8: Velocity and shear stress distribution of the long cavity flow at $x=0, y=0$, and $t=500 \mathrm{~s}$.

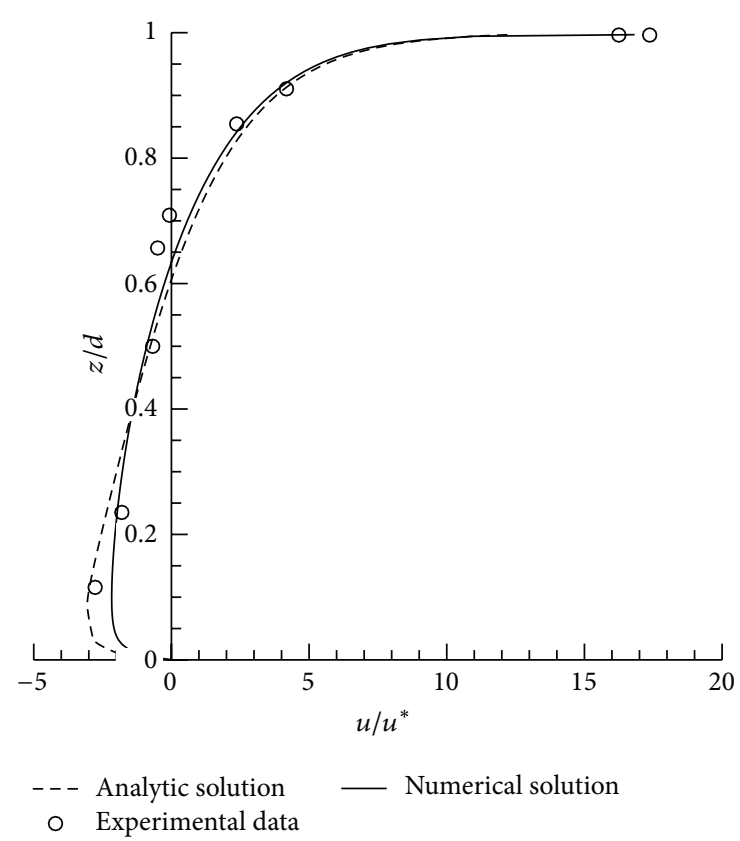

FIgURE 9: Comparison of the computed horizontal velocity at $t=$ $500 \mathrm{~s}$ with experimental data [17] and analytical solution [18] at $x, y=0$ for the long cavity flow.

where $W$ is the Laplace transform of $w$. By the Laplace transform of velocity connection conditions (A1e), (A2a) and (A2b) yield

$$
B(s)=A(s) .
$$

Using (A1f) and (A3), it is derived that

$$
\left(\frac{\mu_{a}}{\sqrt{v_{a}}}+\frac{\mu_{w}}{\sqrt{v_{w}}}\right) A(s)=0,
$$

which requires that $A(s)$ and thus $B(s)$ be zero, that is,

$$
W(y, s) \equiv 0 .
$$

According to Lerch cancellation law [22], (A5) actually implies

$$
w(y, t)=L^{-1}\{W(y, s)\}=0 .
$$

Therefore, problem (22a)-(22f) has a unique solution, which is given as (29).

\section{Acknowledgments}

This work is supported by UTRC and NOAA CREST. In addition, LZZ is funded by China Scholarship Council, and RH is supported by NSF REU Program (Directorate AGU no. 1062934). Partial support for HST and CBJ also comes from China Natural Science Foundation (51239001 and 51179015).

\section{References}

[1] R. Ferrari and C. Wunsch, "Ocean circulation kinetic energy: reservoirs, sources, and sinks," Annual Review of Fluid Mechanics, vol. 41, pp. 253-282, 2009.

[2] A. E. Ozinsky and G. A. Ekama, "Secondary settling tank modelling and design part 1: review of theoretical and practical developments," Water SA, vol. 21, no. 4, pp. 325-332, 1995. 
[3] C. S. Iorio, O. Goncharova, and O. Kabov, "Influence of boundaries on shear-driven flow of liquids in open cavities," Microgravity Science and Technology, vol. 23, no. 4, pp. 373-379, 2011.

[4] R. Abernathey, J. Marshall, and D. Ferreira, "The dependence of southern ocean meridional overturning on wind stress," Journal of Physical Oceanography, vol. 41, pp. 2261-2278, 2011.

[5] P. N. Shankar and M. D. Deshpande, "Fluid mechanics in the driven cavity," Annual Review of Fluid Mechanics, vol. 32, pp. 93-136, 2000.

[6] E. Erturk, "Discussions on driven cavity flow," International Journal for Numerical Methods in Fluids, vol. 60, no. 3, pp. 275294, 2009.

[7] P. A. M. E. Janssen, "Wave-induced stress and the drag of air flow over sea waves," Journal of Physical Oceanography, vol. 19, pp. 745-754, 1989.

[8] P. K. Kundu, Fluid Mechanics, Academic Press, New York, NY, USA, 1990.

[9] H. Schlichting and K. Gersten, Boundary-Layer Theory, Springer, Berlin, Germany, 8th edition, 2000.

[10] M. Devakar and T. K. V. Iyengar, "Stokes' problems for an incompressible couple stress fluid," Nonlinear Analysis: Modelling and Control, vol. 1, no. 2, pp. 181-190, 2008.

[11] C.-M. Liu, "Complete solutions to extended Stokes' problems," Mathematical Problems in Engineering, vol. 2008, Article ID 754262, 18 pages, 2008.

[12] L. Z. Zhang, H. S. Tang, J. P.-Y Maa, and G. Q. Chen, "Exact solutions and analysis for a class of extended Stokes' problems," Discontinuity, Nonlinearity, and Complexity, vol. 2, no. 1, pp. 85102, 2013.

[13] R. Bandelli, K. R. Rajagopal, and G. P. Galdi, "On some unsteady motions of fluids of second grade," Archives of Mechanics, vol. 47, no. 4, pp. 661-676, 1995.

[14] Y. M. Yatim, B. R. Duffy, and S. K. Wilson, "Similarity solutions for unsteady shear-stress-driven flow of Newtonian and powerlaw fluids: slender rivulets and dry patches," Journal of Engineering Mathematics, vol. 73, pp. 53-69, 2012.

[15] N. G. Hadjiconstantinou, "Oscillatory shear-driven gas flows in the transition and free-molecular-flow regimes," Physics of Fluids, vol. 17, no. 10, Article ID 100611, 2005.

[16] H. S. Tang, S. C. Jones, and F. Sotiropoulos, "An oversetgrid method for 3D unsteady incompressible flows," Journal of Computational Physics, vol. 191, pp. 567-600, 2003.

[17] W. D. Baines and D. J. Knapp, "Wind driven water currents," Journal of the Hydraulics Division, vol. 91, no. 2, pp. 205-221, 1965.

[18] R. O. Reid, "Modification of the quadratic Bottom-Stress Law for turbulent channel flow in the presence of surface wind stress," Technical Memorandum, Beach Erosion Board, 1957.

[19] C. Chen, H. Liu, and R. C. Beardsley, "An unstructured grid, finite-volume, three-dimensional, primitive equations ocean model: application to coastal ocean and estuaries," Journal of Atmospheric and Oceanic Technology, vol. 20, no. 1, pp. 159-186, 2003.

[20] L. Xie, K. Wu, L. Pietrafesa, and C. Zhang, "A numerical study of wave-current interaction through surface and bottom stresses: wind-driven circulation in the South Atlantic Bight under uniform winds," Journal of Geophysical Research C, vol. 106, no. 8, pp. 16841-16855, 2001.

[21] "Fluent mannul," ANSYS, Inc., 2010.

[22] J. L. Schiff, The Laplace Transform: Theory and applications, Springer, New York, NY, USA, 1999. 


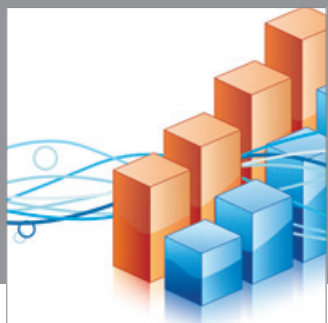

Advances in

Operations Research

mansans

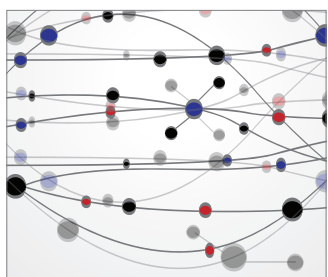

The Scientific World Journal
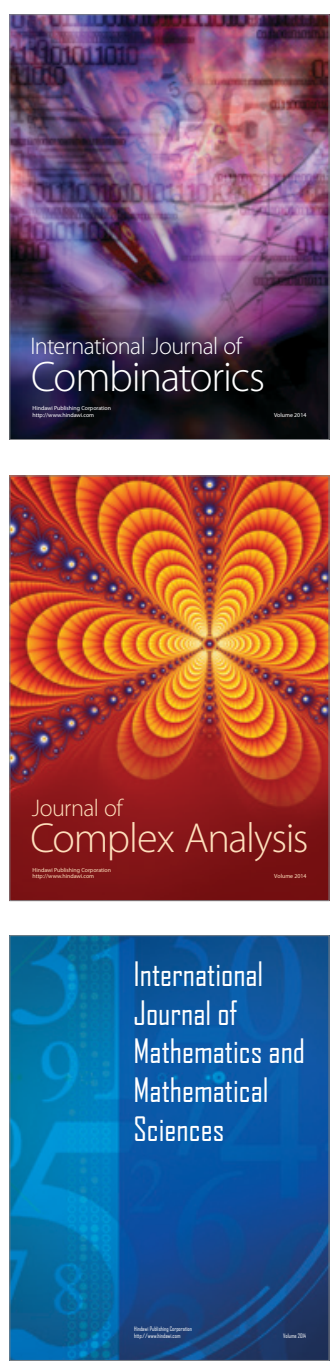
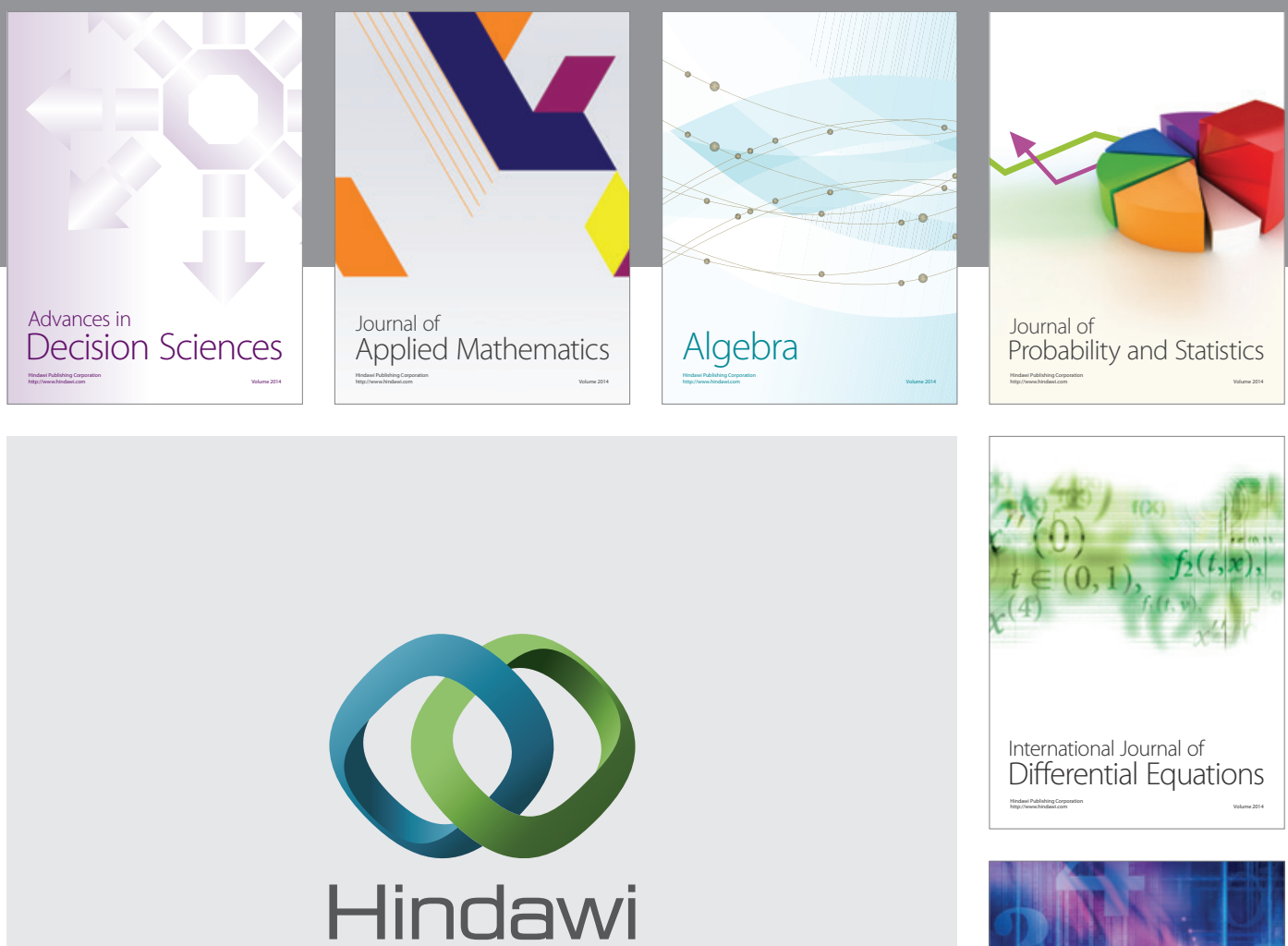

Submit your manuscripts at http://www.hindawi.com
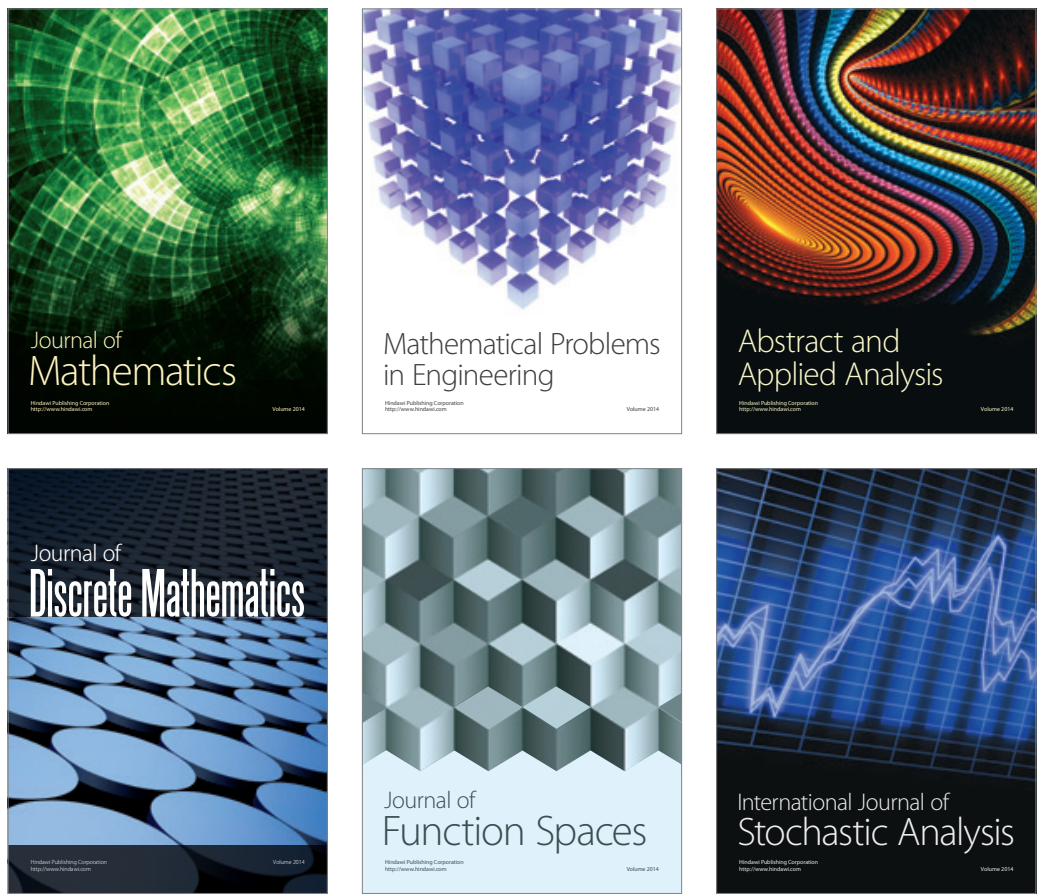

Journal of

Function Spaces

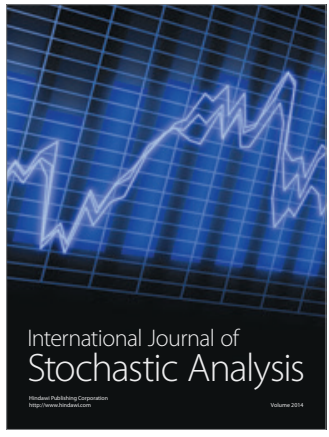

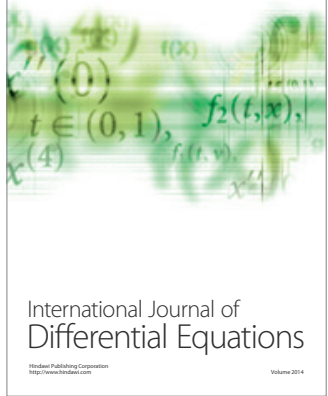
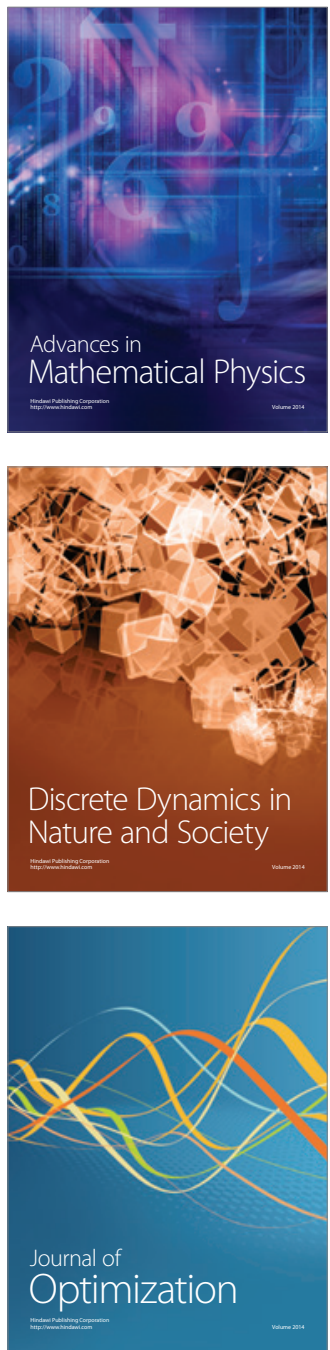